\title{
A New Grating Displacement Measuring Method for Improving Resolution
}

\author{
Lun Shi \\ School of Mechanical Engineering, Shanghai Jiao Tong University, Shanghai, China \\ 200240 \\ shilun@sjtu.edu.cn
}

Keywords: Grating interferometer; phase shift; displacement, ruling engine.

Abstract. A new method for improving resolution of displacement measuring system is developed in this paper. The core parts of the system is a grating interferometer and a phase-shift device. Moving the photoelectric sensor, between the two interference fringes, the signal phase of the interference fringe will be changed. The method leads to higher resolution in theory than that with laser interferometer. Experiments with the method are made in a positioning system and the' results show that the new displacement measuring method will improve the positioning accuracy about $16 \%$.

\section{Introduction}

Grating interferometer is one of the traditional method in displacement measuring system ${ }^{[1,2]}$ with measuring resolution no better than 0.5 micrometer usually. Laser interferometer, with higher measurement resolution, is limited in application sometimes because of its strict requirements on temperature, humidity and so on ${ }^{[3-5]}$. On the other hand, though the resolution of laser interferometer can reach to sub-nanometer, it is limited by the laser wavelength. And coarse/fine dual-stage manipulators are widely applied for long distance movement with high precision ${ }^{[6-9]}$, though they are composed with complicated mechanisms. Therefore, grating interferometer is selected as the study object in this paper. Subdivision method for improving measuring resolution, maybe reach to 0.1 micrometer or even smaller, on grating interferometer is developed widely and the subdivision level is limited by the stripe quality ${ }^{[10]}$. In this paper, a phase shift method is developed and it will lead to higher displacement resolution in sub-nanometer measurement than laser one because of it almost has little limit in theory.

\section{Principle of grating interferometer displacement measuring system}

The grating interferometer displacement measuring system mainly consists of a blazed diffraction grating and a beam splitter with equal optical path, as shown in Fig.1. When the parallel incident light beam, from $\mathrm{D}$ direction, meets the plane $\mathrm{O}-\mathrm{O}$ of the splitter, it will be split into two beams, one is transmitted light beam and another is reflected light beam. When the diffraction grating is in the case of auto-collimation, the two beams will be both diffracted back and will meet together at point $\mathrm{C}$. The interference fringes will appear in the place where the two diffraction beams overlap.

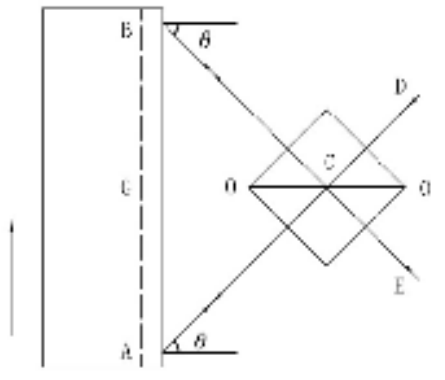

Fig. 1 Schematic of grating interferometer 
The reference diffraction grating is usually mounted on a linear table. When the linear table moves, the interference fringes will be detected and converted into pulse signals by the sensor placed at $\mathrm{E}$ point. When the linear table shifts $\Delta x$ along the direction shown in Fig. 1, the phase variation of the two coherent light beams is ${ }^{[11]}$ :

$$
\Delta \Phi=4 \pi m \frac{\Delta x}{A}
$$

Where $m$ is the diffraction order and $A$ is the space of the reference grating (for example $1 / 300 \mathrm{~mm}$ ). If $\Delta x=A$ and $m=2$, then $\Delta \Phi=2 \pi \times 4$

Therefore, the equivalent displacement to one fringe is:

$$
a^{\prime}=\frac{A}{2 m}
$$

\section{Interference fringes phase shift method}

Adjusting the light path to be sure that there are only two interference fringes, in the detecting field, shown in Fig 2.

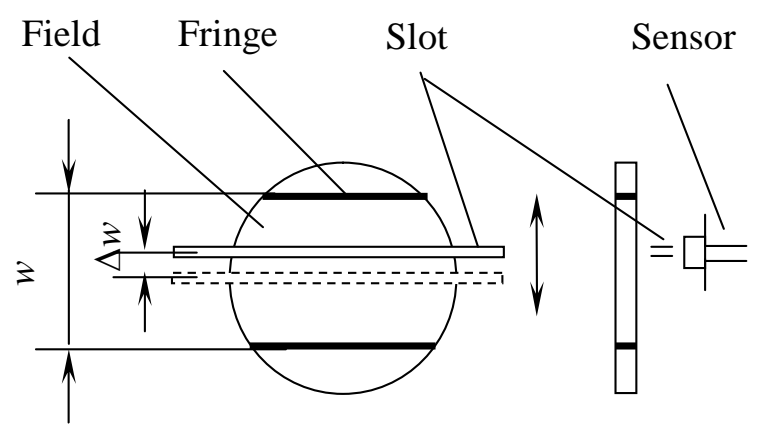

Fig. 2 Phase shift of interference fringe

The $w$ value can be changed by adjusting the interferometer. It is regarded that the fringe phase signal is divided into unlimited parts from $0^{\circ}$ to $360^{\circ}$ in electrical degree and the parts are distributed averagely between the two fringes in theory. Therefore, when we change the position of the photoelectric sensor, the phase of the fringe signal will be changed correspondingly.

The resolution of the method changes with the phase shift value and distance value between the two fringes. In theory the method can get higher resolution than the laser interferometer. The resolution can be expressed as:

$$
d a= \pm \frac{\Delta w}{w} \cdot a^{\prime}
$$

Where, $\Delta w$----- phase shift value

$w$----- distance value between the two fringes

When the positioning table feeds forward, the interference fringes will move up or down. If $a^{\prime}=0.83 \mu m, \Delta w=10 \mu m$ and $w=10 \mathrm{~mm}$, then $d a=0.83 \mathrm{~nm}$.

\section{Experiments}

Some positioning experiments with laser grating interferometer as measuring reference has been made by the way of the stripe phase shift method on a precision positioning system. The photo of the experiments is shown in Fig.3. 
In the experiments above, the grating space of the grating interferometer is $0.83 \mu \mathrm{m}$, with second order of interference fringe $(\mathrm{m}=2)$ and $w=10 \mathrm{~mm}$.

The positioning point is selected at the point of $4 \mathrm{~mm}$ in the experiments. For example, in the $4 \mathrm{~mm}$ experiments, $4000 \mu \mathrm{m} / 0.83 \mu \mathrm{m}=4819.3$ pulse. Therefore, the positioning error of traditional method is $0.25 \mu \mathrm{m}$ in theory. In the experiments, the photo electric sensor will move after the last pulse and the positioning error will be $0.09 \mathrm{~nm}$ in theory. Actually, the error is bigger than $0.09 \mathrm{~nm}$.

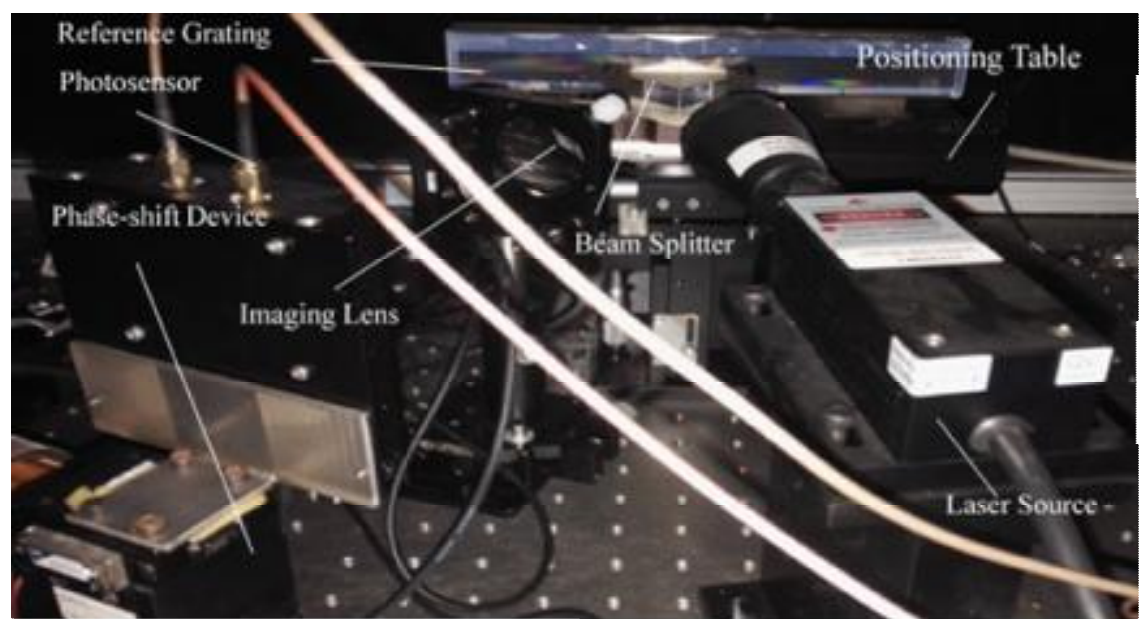

Fig.3 The photo of the experiments

Table 1. Experiments Results of the Positioning with the Phase Shift Method

\begin{tabular}{c|cccc}
\hline & \multicolumn{2}{|c}{ Traditional Method } & \multicolumn{2}{c}{ Phase-shift Method } \\
& Positioning $[\mu \mathrm{m}]$ & Error $[\mathrm{nm}]$ & Positioning $[\mu \mathrm{m}]$ & Error $[\mathrm{nm}]$ \\
\hline 1 & 3999.842 & 158 & 4000.154 & 154 \\
2 & 3999.821 & 179 & 4000.138 & 138 \\
3 & 3999.81 & 183 & 4000.152 & 152 \\
4 & 3999.873 & 127 & 4000.131 & 131 \\
5 & 3999.789 & 211 & 4000.143 & 143 \\
\hline
\end{tabular}

The contrastive results on the positioning experiments with traditional method and new method are shown in the table above. It indicates that the shift method will improve the positioning accuracy about $16 \%$.

This method above can be used in many applications, such as in fabricating varied-line space (VLS) grating in ruling engine. The photograph of the experimental VLS grating ruled with the phase shift method, with space $3.33 \mu \mathrm{m}$ and space increment $20 \mathrm{~nm}$, observed under the atom force microscope (AFM) is shown in Figure 4.

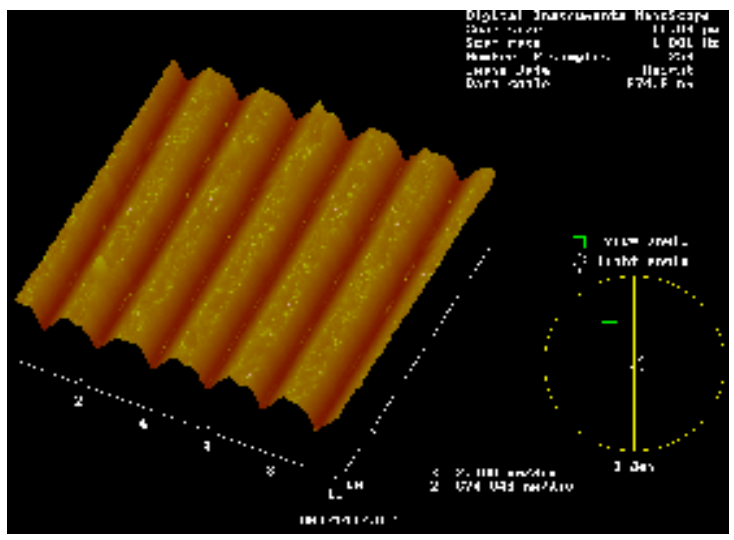


Fig. 4. Photograph of VLS grating under AFM

\section{Conclusion}

Based on fringe phase shifting, a new displacement measuring method, with grating interferometer, is developed. When the signal phase is changed by moving the photoelectric sensor between the two interference fringes in the view field, the displacement equivalent converted from the photoelectric sensor will changed correspondingly. The displacement measurement resolution is higher in theory than that with laser interferometer. The positioning experiments using the new method have been made and the results indicate that it will improve the positioning accuracy about $16 \%$.

\section{Acknowledgements}

This work was financially supported by the NSFC (51275306).

\section{Reference}

[1] HAO D F: Error Transfer Function for Grating Interferometer, Proceedings of SPIE, Vol. 1545 (1991), p. 261.

[2]. Marek Dobosz: Analysis of tolerances in a grating interferometer for high-resolution displacement measurement, Proceedings of SPIE, Vol. 3744 (1999), p. 253.

[3] Valery Kiryanov: Laser nanointerferometry of displacement methods and means of measurement accuracy improvement, Proceedings of SPIE, Vol. 3736 (1999), p. 410.

[4] Yoshioka H, Kuroyama S, Sawano H and Shinno H: Sub-nanometer positioning with a high resolution laser interferometer, Proceedings of the 10th International Conference of the European Society for Precision Engineering and Nanotechnology, Vol. 1 (2010), p. 404.

[5] D. Croft and S. Devasia: High precision stages for micro/nano-lithography, Proceedings of SPIE, Vol. 3225 (1997), p. 68.

[6] Kawashima, Kenji, Arai and etal: Development of coarse/fine dual stage using pneumatically driven bellows actuator and cylinder with air bearings, Precision Engineering, Vol. 34 (2010), p. 526.

[7] Shirinzadeh Bijan and Teoh Pek L: Dual position sensitive diode-based orientation measurement in laser interferometry-based sensing and measurement technique, Proceedings of SPIE, Vol. 4564 (2001), p. 98.

[8] Kwon S, Wan Kyun Chung, and Youm Y: On the coarse/fine dual-stage manipulators with robust perturbation compensator, Proceedings of IEEE, International Conference on Robotics and Automation, Vol. 1 (2001), p. 121.

[9] Kwon SangJoo, Chung Wan Kyun and Youm Youngil: Robust and time-optimal control strategy for coarse/fine dual-stage manipulators, Proceedings of IEEE, International Conference on Robotics and Automation, Vol. 4 (2000), p. 4051.

[10] Wang Wei: Program Controlled Amplifier and Its Typical Application, OPTICS AND PRECISION ENGINEERING, Vol. 6 (1998), p. 89.

[11] Shi L, Hao D F and Qi X D: High Precision Photoelectric Diffraction Grating Ruling Engine, Chinese Journal of Scientific Instrument, Vol. 4 (2001), p. 103. 